Thus every neighbourhood of $x$ contains points $y$ such that $y \in B$ and $P(y, A)=0$. Hence

$$
0 \leqq P\left(y, A_{1}\right) \leqq P(y, A)=0 .
$$

But this contradicts the continuity of $P(x, A)$.

\title{
BIBLIOGRAPHY
}

1. K. L. Chung, Markov chains, Springer, Berlin, 1960.

2. N. Dunford and J. Schwartz, Linear operators, Interscience, New York, 1958.

3. P. R. Halmos, Measure theory, Van Nostrand, New York, 1950.

4. S. Kakutani and K. Yosida, Operator theoretical treatment of Markov processes and mean ergodic theorem, Ann. of Math. (2), 42 (1941), 188-228.

5. J. L. Kelley, General topology, Van Nostrand, New York, 1955.

HEBREW UNIVERSITY

\section{DARBOUX FUNCTIONS OF BAIRE CLASS ONE AND DERIVATIVES}

\section{J. NEUGEBAUER ${ }^{1}$}

Introduction. Let $I_{0}=[0,1]$ and let $R$ be the reals. Let $B_{1}$ be the class of functions $f: I_{0} \rightarrow R$ of Baire type at most one, and denote by $D$ the class of functions $f: I_{0} \rightarrow R$ which possess the Darboux property, i.e., take connected sets into connected sets. The class $B_{1} \cap D$ is abbreviated by $\left(B_{1}, D\right)$. If $\Delta$ is the class of functions $f: I_{0} \rightarrow R$ which are derivatives, then we have the well-known relation $\Delta C\left(B_{1}, D\right)$. It is of interest to have characterizations for the classes $\Delta$ and $\left(B_{1}, D\right)$. In this paper two characterizations of $\left(B_{1}, D\right)$ are given as well as a characterization of $\Delta$. This characterization together with one characterization of $\left(B_{1}, D\right)$ provides a measurement by how much a function in $\left(B_{1}, D\right)$ may fail to be in $\Delta$.

Throughout the paper we will use the following notation. For $A \subset I_{0}, A^{0}$ is the interior of $A$ relative to $I_{0}, \bar{A}$ stands for the closure of $A$, and $|A|$ denotes the Lebesgue measure of $A$.

First characterization of $\left(B_{1}, D\right)$. We have occasion to use the following characterizations of $B_{1}$. (1) $f \in B_{1}$ if and only if for each $a \in R$ the sets $\{x: f(x) \geqq a\},\{x: f(x) \leqq a\}$ are $G_{\delta} ;(2) f \in B_{1}$ if and only if every perfect subset $P$ of $I_{0}$ has a point of continuity of $f \mid P$ ( $f$ restricted to $P$ ) [3].

Presented to the Society January 25, 1962; received by the editors January 8, 1962.

${ }^{1}$ Supported by National Science Foundation Grant G-18920. 
TheOREM 1. $f \in\left(B_{1}, D\right)$ if and only if for each $a \in R$, the sets $\{x: f(x) \geqq a\},\{x: f(x) \leqq a\}$ are $G_{\delta}$ with compact components.

Proof. Let $f \in\left(B_{1}, D\right)$, and let $a \in R$. By (1) we only need to show that the components of $\{x: f(x) \geqq a\},\{x: f(x) \leqq a\}$ are compact. Let $Q$ be a component of $E=\{x: f(x) \geqq a\}$. We may suppose that $Q$ is a nondegenerate interval with endpoints $\alpha<\beta$. If $f(\alpha)<a$, then the set $f([\alpha, \beta))$ would be disconnected, contradicting $f \in D$. Therefore, $\alpha \in E$ and similarly $\beta \in E$. Consequently, $Q=[\alpha, \beta]$, and the proof of the necessity is complete.

For the sufficiency we observe first that $f \in B_{1}$ by (1). If we deny $f \in D$, we have an interval $[\alpha, \beta] \subset I_{0}$ such that $f([\alpha, \beta])$ is not connected. For notational simplification we may assume that $[\alpha, \beta]=I_{0}$. There exists $a \in R$ such that the sets $E_{1}=\{x: f(x) \leqq a\}$, $E_{2}=\{x: f(x) \geqq a\}$ satisfy $I_{0}=E_{1} \cup E_{2}, E_{1} \neq \varnothing, E_{2} \neq \varnothing$, and $E_{1} \cap E_{2}$ $=\varnothing$. Let $\{Q\}_{1}$ be the collection of components of $E_{1}$ which are nondegenerate, and let $\{Q\}_{2}$ be defined analogously. Let $\{Q\}=\{Q\}_{1}$ $\cup\{Q\}_{2}$. By hypothesis, each $Q \in\{Q\}$ is compact, and two distinct components in $\{Q\}$ are disjoint. Hence the set $P=I_{0}-\cup Q^{0}$ is perfect, where the union is extended over all $Q \in\{Q\}$. By (2) there is a point $x_{0} \in P$ at which $f \mid P$ is continuous. Since $I_{0}=E_{1} \cup E_{2}$, we may assume that $x_{0} \in E_{1}$, and since $E_{1} \cap E_{2}=\varnothing$, we infer that $f\left(x_{0}\right)<a$. Therefore, there is a $\delta>0$ such that $I_{\delta} \cap P \subset E_{1}$, where $I_{\delta}$ $=\left[x_{0}-\delta, x_{0}+\delta\right] \cap I_{0}$.

Let $Q \in\{Q\}_{2}$. We will prove that $I_{\delta} \cap Q=\varnothing$. For, if $I_{\delta} \cap Q \neq \varnothing$, there is an endpoint $\gamma$ of $Q$ which is in $I_{\delta}$. Since $\gamma \in E_{2} \cap P$, this contradicts $I_{\delta} \cap P \subset E_{1}$. Since every $x \in E_{2}-\cup Q^{0}$ is in $P$, where the union is extended over all $Q \in\{Q\}_{2}$, we infer that $I_{\delta} \cap E_{2}=\varnothing$, and hence $I_{\delta} \subset E_{1}$. There is then $Q \in\{Q\}_{1}$ such that $I_{\delta} \subset Q$, and thus $x_{0} \in Q^{0}$, contradicting $x_{0} \in P$. This completes the proof.

REMARK. In the necessity of Theorem 1 we have actually proved that for every $f \in D$ the sets $\{x: f(x) \geqq a\},\{x: f(x) \leqq a\}$ have compact components. Without the condition that these sets be $G_{\delta}$ the converse is not true as the example $f(x)=1, x$ rational, $f(x)=0, x$ irrational, shows.

Applications. Theorem 1 can be applied to show that various derivatives are in $\left(B_{1}, D\right)$. As an application, we will consider approximate derivatives and $n$th Peano derivatives. For the properties of approximate derivatives that will be used see [2], and for those of the $n$th Peano derivative see [4]. In these papers it has been shown that approximate derivatives and $n$th Peano derivatives are in $\left(B_{1}, D\right)$. We will show that Theorem 1 provides a very simple proof of this fact. 
Theorem 2. If $f: I_{0} \rightarrow R$ is an approximate derivative or nth Peano derivative, then $f \in\left(B_{1}, D\right)$.

Proof. It is known that $f \in B_{1}[2 ; 4]$. If $Q$ is a component of $\{x: f(x) \geqq a\}$, simple arguments used in the above mentioned papers show that $\bar{Q} \subset\{x: f(x) \geqq a\}$, and thus the components of $\{x: f(x) \geqq a\}$ are compact.

Theorem 1 can be applied to provide a simple proof of a characterization of $\left(B_{1}, D\right)$ due to Zahorski [5]. Let $f: I_{0} \rightarrow R$, and let for $a \in R$, $E_{a}=\{x: f(x)>a\}, B_{a}=\{x: f(x)<a\}$.

Definition (ZAHORSKI). $f \in M_{0}\left(f \in M_{1}\right)$ if and only if for each $a \in R$ the sets $E_{a}, B_{a}$ are $F_{\sigma}$ and each point of $E_{a}, B_{a}$ is a bilateral point of accumulation (condensation) of $E_{a}, B_{a}$, respectively.

Theorem 3. $M_{0}=M_{1}=\left(B_{1}, D\right)$.

Proof. The inclusion $M_{1} \subset M_{0}$ is obvious, and $\left(B_{1}, D\right) \subset M_{1}$ is easy to prove. It suffices to show that $M_{0} \subset\left(B_{1}, D\right)$. Since $f \in M_{0}$ implies that $f \in B_{1}$, for each $a \in R$, the sets $\{x: f(x) \geqq a\},\{x: f(x) \leqq a\}$ are $G_{\delta}$. Let $Q$ be a component of $E=\{x: f(x) \geqq a\}$. We may assume that $Q$ is an interval with endpoints $\alpha<\beta$. If $f(\alpha)<a$, the point $\alpha$ would not be a right point of accumulation of $\{x: f(x)<a\}$. Hence $f(\alpha) \geqq a$, and similarly, $f(\beta) \geqq a$. Thus $Q=\bar{Q}$, and by Theorem 1 the proof is complete.

Second characterization of $\left(B_{1}, D\right)$. Let $\{I\}$ be the collection of all nondegenerate compact subintervals of $I_{0}$. We shall use the notation $I \rightarrow x$ to denote $x \in I$ and $|I| \rightarrow 0$. Following Gleyzal [1] we say that an interval function $\phi:\{I\} \rightarrow R$ converges on $I_{0}$ if and only if for each $x \in I_{0}, \lim _{I \rightarrow x} \phi(I)$ exists. In [1], Gleyzal proved the following characterization of $B_{1}: f \in B_{1}$ if and only if $f$ is the limit of a convergent interval function.

Definition. A function $f: I_{0} \rightarrow R$ is said to possess property $\left(C_{1}\right)$ if and only if for each $I \in\{I\}$ there exists $x_{I} \in I^{0}$ such that $I \rightarrow x$ implies that $f\left(x_{I}\right) \rightarrow f(x)$, for each $x \in I_{0}$.

REMARK. A comparison with ordinary continuity may clarify the condition $\left(C_{1}\right)$. A function $f: I_{0} \rightarrow R$ is continuous on $I_{0}$ if and only if $\left(^{*}\right) I \rightarrow x, x_{I} \in I^{0}$ implies $f\left(x_{I}\right) \rightarrow f(x)$, for each $x \in I$. Condition $\left(C_{1}\right)$ is a formulation of the weakest version of $\left(^{*}\right)$ in the sense that we only require that in each $I^{0}$ there is a point $x_{I}$ so that $I \rightarrow x$ implies $f\left(x_{I}\right)$ $\rightarrow f(x)$.

TheORem 4. $f \in\left(B_{1}, D\right)$ if and only if $f$ has property $\left(C_{1}\right)$. 
Proof. First, suppose that $f$ has property $\left(C_{1}\right)$. If $\phi(I)=f\left(x_{I}\right)$, then $\phi(I) \rightarrow f(x)$ as $I \rightarrow x$. Consequently, by Gleyzal's theorem, $f \in B_{1}$. In order to show that $f \in D$, we only need to show, by Theorem 1 , that the sets $\{x: f(x) \geqq a\},\{x: f(x) \leqq a\}$ have compact components. Let $Q$ be a component of $E=\{x: f(x) \geqq a\}$. We may assume that $Q$ is a nondegenerate interval with endpoints $\alpha<\beta$. Let $x_{n} \in(\alpha, \beta)$ with $x_{n} \rightarrow \alpha$, and let $I_{n}=\left[\alpha, x_{n}\right]$. Then $I_{n} \rightarrow \alpha$, and thus $f\left(x_{I_{n}}\right) \rightarrow f(\alpha)$. Since $x_{I_{n}} \in I_{n}^{0} \subset Q$, we have $f\left(x_{I_{n}}\right) \geqq a$, and thus $f(\alpha) \geqq a$. Similarly, $f(\beta) \geqq a$, and hence $Q=\bar{Q}$.

The necessity is more complicated. We assume that $f \in\left(B_{1}, D\right)$. Since $f \in B_{1}$, there is a sequence of continuous functions $f_{n}: I_{0} \rightarrow R$ such that $f_{n} \rightarrow f$ on $I_{0}$. For each $n$, select a finite number of intervals $I_{n 1}, \cdots, I_{n j_{n}}$ in $\{I\}$ such that $I_{0}=I_{n 1}^{0} \cup \cdots \cup I_{n j_{n}}^{0},\left|I_{n k}\right|<1 / n$, $k=1, \cdots, j_{n}$, and the saltus of $f_{n}$ on each $I_{n k}$ is $<1 / n$. Define $\{I\}_{1}=\left\{I_{n k}, k=1, \cdots, j_{n} ; n=1,2, \cdots\right\}$. We note that $\{I\}_{1}$ covers $I_{0}$ in the sense of Vitali.

Let $\{I\}_{2}$ be the collection of all $I \in\{I\}$ for which there exists $J \in\{I\}_{1}$ such that $I \subset J$, and let $\{I\}_{3}$ be the collection of the remaining intervals. For $I \in\{I\}_{2}$, let $n_{0}$ be the largest integer for which there exists $k, 1 \leqq k \leqq j_{n_{0}}$, such that $I \subset I_{n_{0} k}$. Among the $k$ 's for which $I \subset I_{n_{0} k}$ select one $k_{0}$, and call $\left(n_{0}, k_{0}\right)$ the pair associated with $I$.

Let $I \in\{I\}_{2}$ and let $(n, k)$ be the pair associated with $I$. Since $f \in D, \overline{f(I)}$ is a closed interval, and since $f_{n}$ is continuous, $f_{n}\left(I_{n k}\right)$ is a compact interval. Hence there are points $y_{n k} \in I_{n k}, \xi_{I} \in \overline{f(I)}$ such that

$$
\left|f_{n}\left(y_{n k}\right)-\xi_{I}\right|=\operatorname{dist}\left[f_{n}\left(I_{n k}\right), f(I)\right] \text {. }
$$

Since $f \in D$, we can choose $x_{I} \in I^{0}$ such that $\left|\xi_{I}-f\left(x_{I}\right)\right|<1 / n$. If $I \in\{I\}_{3}$, select any $x_{I} \in I^{0}$. For each $I \in\{I\}$ we have now chosen a point $x_{I} \in I^{0}$. We will verify that $f\left(x_{I}\right) \rightarrow f(x)$ if $I \rightarrow x$. We will proceed in several steps.

STEP 1. Let $I \in\{I\}_{2}$ and let $(n, k)$ be the pair associated with $I$. Then $I \subset I_{n k}$. We define $\phi_{1}(I)=f_{n}\left(y_{n k}\right)$. We will show that $\phi_{1}$ converges to $f$ on $I_{0}$. This part of the proof is essentially a reproduction of Gleyzal's argument [1] with some modifications. Let $\xi \in I_{0}$ and let $\left\{I_{j}\right\} \subset\{I\}_{2}$ be a sequence with $I_{j} \rightarrow \xi$. For each $n$, there is a $j_{n}>0$ such that for $j>j_{n}, I_{j} \subset I_{n k}$ for some $k$. Hence, for $j>j_{n},\left|I_{n_{j} k_{j}}\right|<1 / n$, where $\left(n_{j}, k_{j}\right)$ is the pair associated with $I_{j}$. Since $n_{j} \geqq n$, we have that $n_{j} \rightarrow \infty$ and $I_{n_{j} k_{j}} \rightarrow \xi$ as $j \rightarrow \infty$.

Let $p$ be a positive integer. There is $l>0$ such that $\left|f_{n}(\xi)-f(\xi)\right|$ $<1 / p$ for $n>l$. There is $l^{\prime}>0$ such that $j>l^{\prime}$ implies that $n_{j}>\max (p, l)$. 
Let $j>\max \left(l^{\prime}, l\right)$. Then

$$
\begin{aligned}
\left|\phi_{1}\left(I_{j}\right)-f(\xi)\right| & =\left|f_{n_{j}}\left(y_{n_{j} k_{j}}\right)-f(\xi)\right| \\
& \leqq\left|f_{n_{j}}\left(y_{n_{j} k_{j}}\right)-f_{n_{j}}(\xi)\right|+\left|f_{n_{j}}(\xi)-f(\xi)\right| \\
& <\frac{1}{n_{j}}+\frac{1}{p}<\frac{2}{p} .
\end{aligned}
$$

Hence $\phi_{1}\left(I_{j}\right) \rightarrow f(\xi)$.

SteP 2. For $I \in\{I\}$, let $\phi(I)=f\left(x_{I}\right)$, where $x_{I} \in I^{0}$ is the point chosen above. We will prove that $\phi$ converges to $f$ on $I_{0}$. Let $\xi \in I_{0}$ and let $\left\{I_{j}\right\} \subset\{I\}$ be a sequence with $I_{j} \rightarrow \xi$. There is $j_{1}>0$ such that $j>j_{1}$ implies $I_{j} \subset I_{1 k}$ for some $k$. Hence for $j>j_{1}, I_{j} \in\{I\}_{2}$. Consequently, by step $1, \phi_{1}\left(I_{j}\right) \rightarrow f(\xi)$. For $j>j_{1}$, let $\left(n_{j}, k_{j}\right)$ be the pair associated with $I_{j}$. Then $I_{j} \subset I_{n_{j} k_{j}}, I_{n_{j} k_{j}} \rightarrow \xi$, and $\phi_{1}\left(I_{j}\right)=f_{n_{j}}\left(y_{n_{j} k_{j}}\right)$. Thus

$$
\begin{aligned}
\left|\phi\left(I_{j}\right)-\phi_{1}\left(I_{j}\right)\right| & =\left|f\left(x_{I_{j}}\right)-f_{n_{j}}\left(y_{n_{j} k_{j}}\right)\right| \\
& \leqq\left|f\left(x_{I_{j}}\right)-\xi_{I_{j}}\right|+\left|\xi_{I_{j}}-f_{n_{j}}\left(y_{n_{j} k_{j}}\right)\right|,
\end{aligned}
$$

where $\xi_{I_{j}}$ has been defined previously. Thus

$$
\left|\phi\left(I_{j}\right)-\phi_{1}\left(I_{j}\right)\right|<\frac{1}{n_{j}}+\operatorname{dist}\left[f\left(I_{j}\right), f_{n_{j}}\left(I_{n_{j} k_{j}}\right)\right] \leqq \frac{1}{n_{j}}+\left|f_{n_{j}}(\xi)-f(\xi)\right| .
$$

Since $f_{n_{j}}(\xi) \rightarrow f(\xi)$ as $j \rightarrow \infty$, we infer that $\left|\phi\left(I_{j}\right)-\phi_{1}\left(I_{j}\right)\right| \rightarrow 0$. Hence $\phi\left(I_{j}\right) \rightarrow f(\xi)$, completing the proof.

Characterization of $\Delta$. As before, let $\{I\}$ be the collection of all nondegenerate compact intervals of $I_{0}$.

Definition. A function $f: I_{0} \rightarrow R$ is said to have property $\left(C_{2}\right)$ if and only if for each $I \in\{I\}$ there is $x_{I} \in I^{0}$ such that

(1) $I \rightarrow x$ implies that $f\left(x_{I}\right) \rightarrow f(x)$, for each $x \in I_{0}$,

(2) if $I=I_{1} \cup I_{2}, I_{1}^{0} \cap I_{2}^{0}=\varnothing$, then

$$
f\left(x_{I}\right)=\frac{f\left(x_{I_{1}}\right)\left|I_{1}\right|+f\left(x_{I_{2}}\right)\left|I_{2}\right|}{\left|I_{1}\right|+\left|I_{2}\right|} .
$$

REMARK. Condition (1) is the same as $\left(C_{1}\right)$. Condition (2) says that $f\left(x_{I}\right)$ is between $f\left(x_{I_{1}}\right)$ and $f\left(x_{I_{2}}\right)$. One may refer to (2) by saying that $f\left(x_{I}\right)$ is mean-valued. We note that (2) is the same as saying that $f\left(x_{I}\right)|I|$ is an additive interval function.

Theorem 5. $f \in \Delta$ if and only if $f$ has property $\left(C_{2}\right)$.

Proof. Suppose $f \in \Delta$. Then there exists $F: I_{0} \rightarrow R$ such that on $I_{0}, F^{\prime}(x)=f(x)$. Let $I=[a, b]$ be in $\{I\}$. Then there exists $x_{I} \in I^{0}$ 
such that $F(a)-F(b)=f\left(x_{I}\right)|I|$. Thus the interval function $f\left(x_{I}\right)|I|$ is additive. If $I_{j} \rightarrow x, I_{j}=\left[a_{j}, b_{j}\right]$, then

$$
f\left(x_{I_{j}}\right)=\frac{F\left(b_{j}\right)-F\left(a_{j}\right)}{b_{j}-a_{j}} \rightarrow f(x) .
$$

Conversely, assume that $f$ has property $\left(C_{2}\right)$. Then, for each $I \in\{I\}$ there is $x_{I} \in I^{0}$ with the properties (1) and (2). Define $F(0)=0$ and for $0<x \leqq 1$, define $F(x)=f\left(x_{I}\right)|I|$, where $I=[0, x]$. Since $f\left(x_{I}\right)|I|$ is an additive interval function, it follows that for $I=[a, b]$,

$$
F(b)-F(a)=f\left(x_{I}\right)(b-a) .
$$

Let $x \in I_{0}$ and let $\left\{I_{j}\right\} \subset\{I\}$ be a sequence with $I_{j} \rightarrow x$, and set $I_{j}=\left[a_{j}, b_{j}\right]$. Then, since $f\left(x_{I_{j}}\right) \rightarrow f(x)$, we infer that

$$
\frac{F\left(b_{j}\right)-F\left(a_{j}\right)}{b_{j}-a_{j}} \rightarrow f(x) .
$$

Thus $F^{\prime}(x)=f(x)$, and the proof is complete.

REMARK. Comparing Theorem 5 with Theorem 4, we observe that the condition $(2)$ in $\left(C_{2}\right)$ is precisely the property by which a Darboux function of Baire class one may fail to be a derivative.

It is possible to rewrite Theorem 5 in a slightly different form. An interval function $\phi:\{I\} \rightarrow R$ will be termed mean-valued if and only if $\phi(I)|I|$ is additive.

THEOREM 6. $f \in \Delta$ if and only if $f$ is the limit of a convergent and meanvalued interval function.

The proof is the same as the one above.

\section{BIBLIOGRAPHY}

1. A. Gleyzal, Interval functions, Duke Math. J. 8 (1941), 223-230.

2. C. Goffman and C. J. Neugebauer, On approximate derivatives, Proc. Amer. Math. Soc. 11 (1960), 962-966.

3. I. P. Natanson, Theorie der Funktionen einer reellen Veränderlichen, AkademieVerlag, Berlin, 1954.

4. H. W. Oliver, The exact Peano derivative, Trans. Amer. Math. Soc. 76 (1954), 444-456.

5. Z. Zahorski, Sur la première dérivee, Trans. Amer. Math. Soc. 69 (1950), 1-54.

Purdue University 